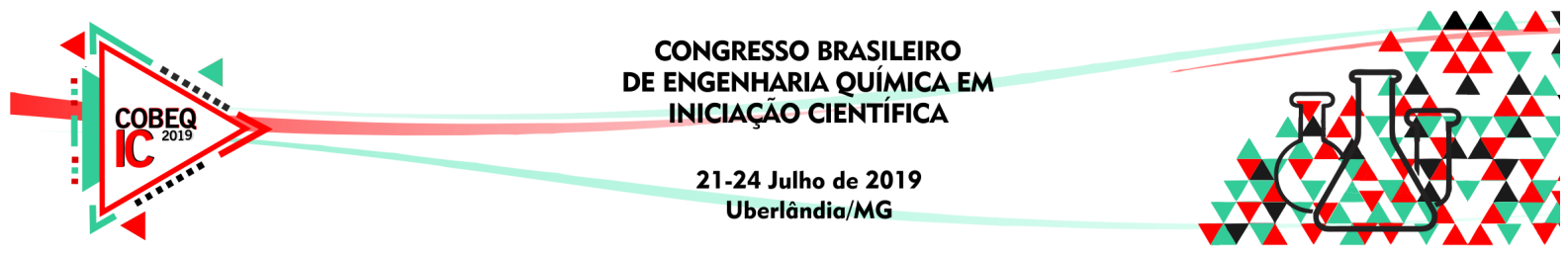

\title{
ESTUDO DA SECAGEM DE FOLHAS DE NIM (Azadirachta indica) POR IRRADIAÇ̃̃O INFRAVERMELHA
}

\author{
L. A. MARQUES JÚNIOR ${ }^{1 *}$; E. D. T. PARCKERT ${ }^{2}$; A. C. SOUZA ${ }^{2,3}$ \\ ${ }^{1}$ Universidade Federal do Triângulo Mineiro, Departamento de Engenharia Química \\ ${ }^{2}$ Universidade Federal do Triângulo Mineiro, Programa de Pós-Graduação Interdisciplinar \\ em Biociências Aplicadas \\ ${ }^{3}$ Universidade Federal do Triângulo Mineiro, Departamento de Engenharia de Alimentos \\ *E-mail: luizmarques05@gmail.com
}

\begin{abstract}
RESUMO - A árvore de nim desperta grande interesse para a comunidade científica, visto que seus extratos são usados na agricultura, pecuária e medicina. O processamento de suas folhas requer condições bem estabelecidas de secagem para evitar a decomposição dos seus compostos bioativos, bem como impedir sua degradação durante o período de armazenamento. Visando expandir os conhecimentos sobre essa planta, o principal objetivo desta pesquisa foi estudar as curvas de cinética de secagem das folhas da árvore nim usando aquecimento por irradiação infravermelha. Dados experimentais de porcentagem de água removida em relação ao tempo foram utilizados para obtenção de curvas de razão de umidade em função do tempo de secagem. Usando o software Origin $\AA$, treze modelos matemáticos empíricos foram ajustados aos dados experimentais para avaliação do melhor modelo de ajuste preditivo. A maioria dos modelos estudados mostraram-se apropriados para descrever a cinética de secagem das folhas de nim nas temperaturas de $60^{\circ} \mathrm{C}, 65^{\circ} \mathrm{C}$ e $70^{\circ} \mathrm{C}$, visto que foram obtidos valores adequados de coeficiente de determinação, módulo de desvio médio relativo e erro médio estimado.
\end{abstract}

\section{INTRODUÇÃO}

Nomeada por Adrien Henri Laurent de Jussieu, em 1830, como Azadirachta indica, a planta nim, também conhecida como neem, nime ou margosa, vem sendo utilizada há séculos no controle de insetos, pragas, nematoides, alguns fungos, bactérias e vírus, na medicina humana e animal, na fabricação de cosméticos, como madeira de lei, adubo e também aplicada às práticas de paisagismo, devido à alta resistência apresentada à seca. (Schmutterer, 1995). Segundo a Agência Nacional de Vigilância Sanitária, azadiractina é o termo aplicado a um grupo de compostos limonoides com ação inseticida, extraídos de sementes da árvore nim. O grupo não é completamente identificável e quantificável e, assim, a azadiractina A refere-se ao principal composto do grupo, sendo utilizada para identificação e quantificação do princípio ativo (Brasil, 2013).

A azadiractina A, encontrada em sua maioria nas sementes do nim, é solúvel em água, mas pode ser eficientemente obtida por extração metanólica. É especialmente instável em meios ácidos e alcalinos, em altas temperaturas, em presença de luz e de umidade. Desta 


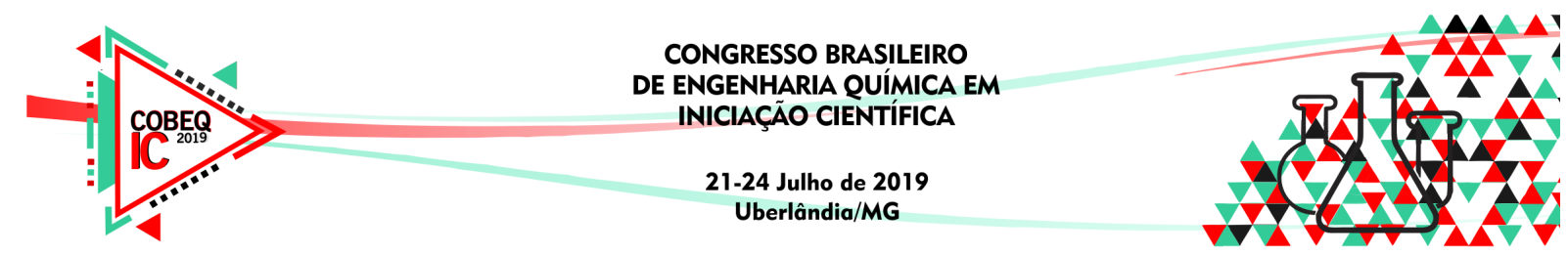

forma, faz-se necessário processar adequadamente os derivados da planta, no sentido de preservar esta substância (Martinez, 2002).

Uma das contribuições da engenharia para o beneficiamento das folhas de Azadirachta indica pode ser dada pela operação de secagem, que traz inúmeras vantagens para a obtenção de um melhor produto após o processamento, pois proporciona boas condições de armazenamento, garantindo estabilidade microbiana, química e física para possível produção de produtos. De acordo com Garcia et al. (2004), a operação de secagem é fundamental no sistema de produção, porque reduz sua a umidade, permitindo assim a conservação de sua qualidade fisiológica no armazenamento, bem como possibilita a antecipação da colheita, evitando perdas de natureza diversa. Sendo assim, o estudo da conservação da folha de nim por desidratação é altamente viável pois a aplicabilidade de seus componentes em forma de pó ou de folhas desidratadas é muito maior do que da folha in natura, devido a facilidade no transporte, no armazenamento e na diluição em solventes para preparo de soluções.

Com base nisso, o objetivo geral da proposta foi proceder à secagem de folhas de nim (Azadirachta indica) em equipamento de aquecimento por irradiação infravermelha, estudando a cinética do processo e a influência da temperatura da irradiação. Foram escolhidos os modelos matemáticos que melhor ajustaram os dados experimentais, usando como parâmetros de escolha o coeficiente de determinação $\left(R^{2}\right)$, o módulo de desvio médio relativo $(P)$ e o erro médio estimado $(S E)$.

\section{MATERIAIS E MÉTODOS}

Foram utilizadas folhas de nim provenientes de árvores do município de Uberaba (Minas Gerais), colhidas entre setembro e março, período de maior folhagem das árvores, e um equipamento medidor de umidade com aquecimento por irradiação infravermelha (modelo IV 2500, marca Gehaka). As seguintes etapas foram executadas: colheita, seleção e limpeza, trituração, secagem por irradiação infravermelha e acondicionamento.

A etapa de secagem foi realizada em triplicata, sob aquecimento por irradiação infravermelha, em amostras de massa conhecida de folhas de nim in natura. Três diferentes temperaturas foram utilizadas: $60^{\circ} \mathrm{C}, 65^{\circ} \mathrm{C}$ e $70^{\circ} \mathrm{C}$. O equipamento de aquecimento por irradiação infravermelha apresenta instantaneamente em seu display dados referentes à quantidade de umidade retirada da amostra em função do tempo. Sendo assim, curvas de razão de umidade $(R U)$ foram graficadas em função do tempo $(t)$, para avaliação da cinética de secagem do produto.

Usando o software Origin ${ }^{\circledR}$, treze modelos matemáticos empíricos foram usados para ajuste dos dados experimentais, sendo eles: Lewis (Equação 1), Page (Equação 2), Brooker (Equação 3), Page Modificado (Equação 4), Thompson (Equação 5), Logarítmico (Equação 6), Henderson \& Pabis Modificado (Equação 7), Midilli (Equação 8), Verna (Equação 9), Dois Termos (Equação 10), Peleg (Equação 11), Silva (Equação 12) e Wang \& Singh (Equação 13). A razão de umidade $(R U)$ expressa a relação entre as diferenças da umidade em cada tempo $(M)$ e da umidade inicial $\left(M_{0}\right)$ com a umidade de equilíbrio $\left(M_{e q}\right)$ da amostra, sendo essa umidade de equilíbrio considerada como a umidade final obtida após obter massa constante (Equação 14). 


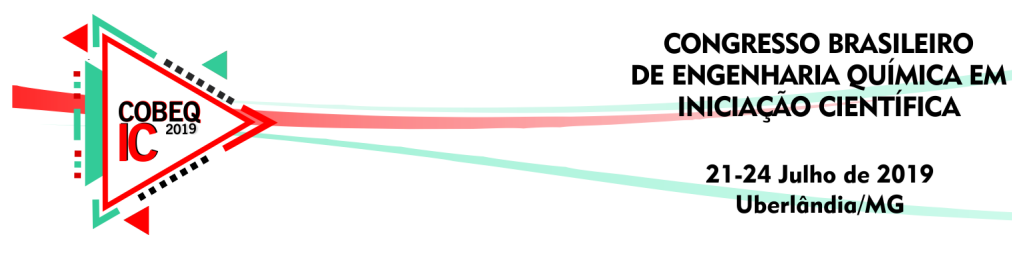

$$
\begin{aligned}
& R U=e^{-k t} \\
& R U=e^{-k t^{n}} \\
& R U=A e^{-k t} \\
& R U=e^{-(k t)^{n}} \\
& R U=a \ln (t)+b \ln (t) \\
& R U=a e^{-k t}+b \\
& R U=a e^{-k t}+b e^{-g t}+c e^{-h t} \\
& R U=a e^{-k t^{n}}+b t \\
& R U=a e^{-k t}+(1-a) e^{-g t} \\
& R U=a e^{-k t}+b e^{-g t} \\
& R U=1-\frac{t}{a+b t} \\
& R U=e^{-a t}-b \sqrt{t} \\
& R U=1+a t+b t^{2} \\
& R U=\frac{\left(M-M_{e q}\right)}{\left(M_{o}-M_{e q}\right)}
\end{aligned}
$$

\section{RESULTADOS E DISCUSSÃO}

Durante a secagem das folhas de nim em equipamento de aquecimento por irradiação infravermelha, observou-se que o aumento da temperatura promoveu uma redução no tempo necessário para a remoção de água do produto, fenômeno observado por diversos pesquisadores trabalhando com outras espécies de plantas medicinais (Gonelli et al., 2014; Evin, 2012; Prates et al., 2012). Para as folhas de nim atingirem a umidade de equilíbrio foram necessários $3120 \mathrm{~s}$ a $60^{\circ} \mathrm{C}, 2580 \mathrm{~s} \mathrm{a} 65^{\circ} \mathrm{C}$ e $2280 \mathrm{~s} \mathrm{a} 70^{\circ} \mathrm{C}$.

Com relação à umidade de equilíbrio, após processo de secagem por aquecimento infravermelho, obteve-se os seguintes valores: 0,0299 $\mathrm{g}_{\text {água }} / \mathrm{g}_{\text {mat.seca }}$ para a temperatura de $60^{\circ} \mathrm{C} ; 0,0246 \mathrm{~g}_{\text {água }} / \mathrm{g}_{\text {mat.seca }}$ para $65^{\circ} \mathrm{C}$ e 0,0204 gágua $/ \mathrm{g}_{\text {mat.seca }}$ para $70^{\circ} \mathrm{C}$. Esses dados mostram que a temperatura causou um efeito na dessorção de umidade pelas folhas, pois com o seu aumento da temperatura houve maior perda de umidade pelas folhas de nim. A Figura 1 mostra os dados experimentais de razão de umidade em função do tempo.

Figura 1 - Dados experimentais de razão de umidade em função do tempo obtidos para as folhas de nim desidratadas por irradiação infravermelha nas temperaturas $60^{\circ} \mathrm{C}, 65^{\circ} \mathrm{C}$ e $70^{\circ} \mathrm{C}$

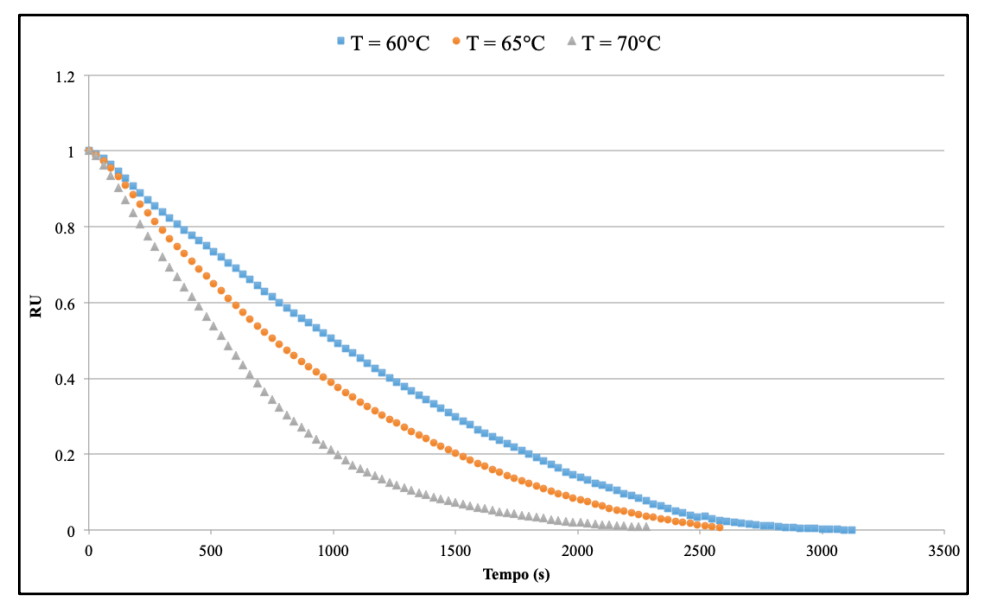

A literatura afirma que o ajuste dos dados é considerado adequado quando: coeficiente de determinação $\left(R^{2}\right)$ é próximo de 1, módulo do desvio médio relativo $(P)$ é inferior a $10 \% \mathrm{e}$ erro médio estimado $(S E)$ é próximo de zero. A modelagem matemática realizada para os dados das curvas de secagem mostrou que os modelos matemáticos de Page Modificado, Henderson \& Pabis Modificado, Midilli, Verna e Wang \& Singh são os mais adequados 

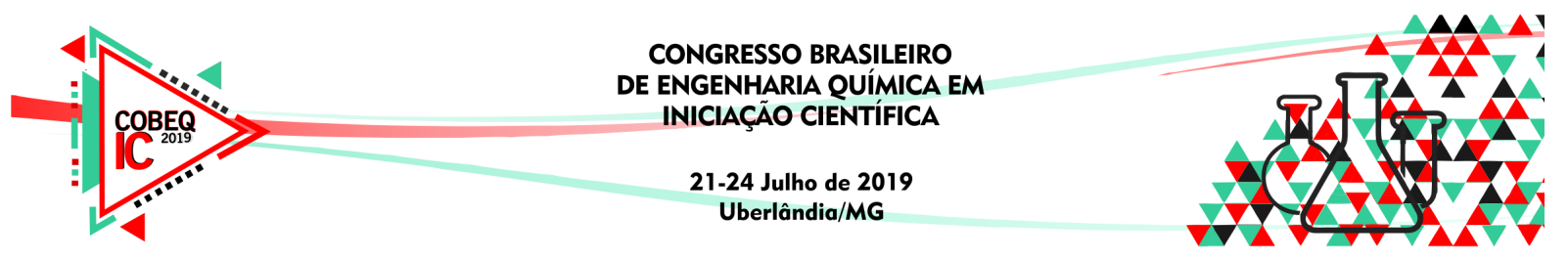

de acordo com os valores encontrados para $R^{2}, P$ e $S E$. As Figuras 2a, $2 \mathrm{~b}$ e $2 \mathrm{c}$ apresentam, respectivamente para as temperaturas de $60{ }^{\circ} \mathrm{C}, 65^{\circ} \mathrm{C}$ e $70{ }^{\circ} \mathrm{C}$, os valores experimentais (pontos) e preditos (linha contínua) de razão de umidade em função do tempo para esses cinco modelos que mostraram ajustes mais satisfatórios. Na Tabela 1 são sumarizados os parâmetros encontrados para os cinco modelos considerados mais ideais.

Figura 2 - Modelos matemáticos ajustados aos dados experimentais nas temperaturas de a) 60 ${ }^{\circ} \mathrm{C}$, b) $65{ }^{\circ} \mathrm{C}$ e c) $70{ }^{\circ} \mathrm{C}$
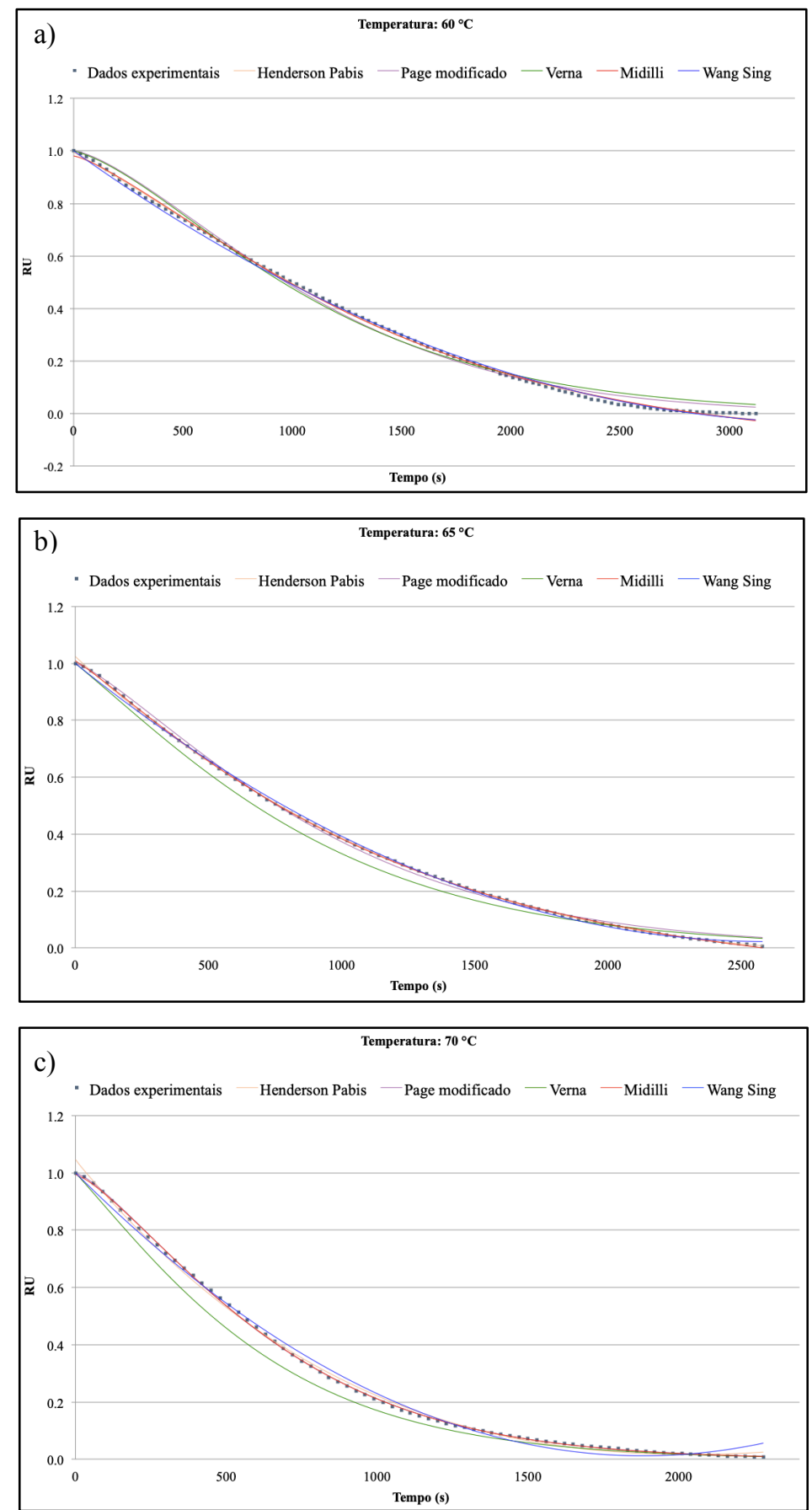


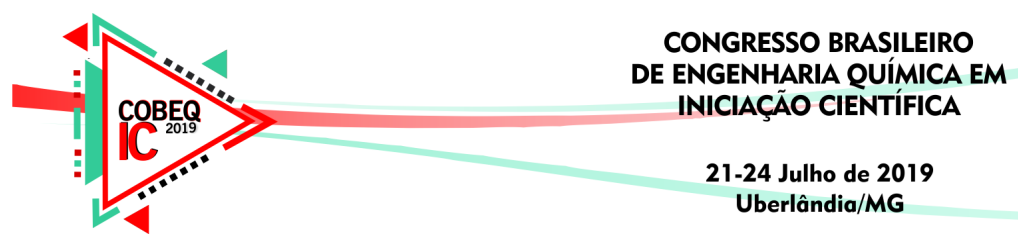

Tabela 1 - Parâmetros ajustados para os modelos matemáticos

\begin{tabular}{|c|c|c|c|c|c|}
\hline Modelo & $\mathbf{T}\left({ }^{\circ} \mathrm{C}\right)$ & Parâmetros & $\mathbf{R}^{2}$ & $\mathbf{P}(\%)$ & SE \\
\hline \multirow{3}{*}{ Page Modificado } & 60 & $\begin{array}{l}\mathrm{k}=0,000797 \\
\mathrm{n}=1,43159\end{array}$ & 0,994 & 1,736 & 0,024 \\
\hline & 65 & $\begin{array}{l}\mathrm{k}=0,0009891 \\
\mathrm{n}=1,27716\end{array}$ & 0,998 & 0,268 & 0,014 \\
\hline & 70 & $\begin{array}{l}\mathrm{k}=0,0014 \\
\mathrm{n}=1,31558\end{array}$ & 0,999 & 0,038 & 0,005 \\
\hline \multirow{3}{*}{$\begin{array}{c}\text { Henderson \& Pabis } \\
\text { Modificado }\end{array}$} & 60 & $\begin{array}{l}\mathrm{a}=-98,94101 \\
\mathrm{~b}=194,03497 \\
\mathrm{c}=-94,09929 \\
\mathrm{k}=0,0008592 \\
\mathrm{~g}=0,000808 \\
\mathrm{~h}=0,000758\end{array}$ & 0,999 & 1,073 & 0,008 \\
\hline & 65 & $\begin{array}{l}\mathrm{a}=21,38647 \\
\mathrm{~b}=-51,65132 \\
\mathrm{c}=31,28844 \\
\mathrm{k}=0,0001198 \\
\mathrm{~g}=0,0001827 \\
\mathrm{~h}=0,00024703\end{array}$ & 0,999 & 0,007 & 0,004 \\
\hline & 70 & $\begin{array}{l}\mathrm{a}=-156,2883 \\
\mathrm{~b}=70,42596 \\
\mathrm{c}=86,90714 \\
\mathrm{k}=0,000260134 \\
\mathrm{~g}=0,000204249 \\
\mathrm{~h}=0,000317012\end{array}$ & 0,998 & 0,169 & 0,012 \\
\hline \multirow{3}{*}{ Midilli } & 60 & $\begin{array}{l}\mathrm{a}=0,98133 \\
\mathrm{~b}=-0,0000273 \\
\mathrm{k}=0,0000729 \\
\mathrm{n}=1,31309\end{array}$ & 0,999 & 1,293 & 0,010 \\
\hline & 65 & $\begin{array}{l}\mathrm{a}=1,00796 \\
\mathrm{~b}=-0,0000278 \\
\mathrm{k}=0,0003451 \\
\mathrm{n}=1,13827\end{array}$ & 0,999 & 0,041 & 0,003 \\
\hline & 70 & $\begin{array}{l}\mathrm{a}=0,99352 \\
\mathrm{~b}=0,000000089 \\
\mathrm{k}=0,000160192 \\
\mathrm{n}=1,32859\end{array}$ & 0,999 & 0,037 & 0,005 \\
\hline \multirow{3}{*}{ Verna } & 60 & $\begin{array}{l}\mathrm{a}=-139,5263 \\
\mathrm{k}=0,00162 \\
\mathrm{~g}=0,00161\end{array}$ & 0,992 & 2,375 & 0,028 \\
\hline & 65 & $\begin{array}{l}\mathrm{a}=-104,97596 \\
\mathrm{k}=0,00183 \\
\mathrm{~g}=0,00182\end{array}$ & 0,997 & 0,280 & 0,035 \\
\hline & 70 & $\begin{array}{l}\mathrm{a}=-76,15501 \\
\mathrm{k}=0,00271 \\
\mathrm{~g}=0,00269\end{array}$ & 0,999 & 0,147 & 0,044 \\
\hline \multirow{3}{*}{ Wang \& Sing } & 60 & $\begin{array}{l}\mathrm{a}=-0,000595 \\
\mathrm{~b}=0,0000001\end{array}$ & 0,999 & 1,185 & 0,011 \\
\hline & 65 & $\begin{array}{l}\mathrm{a}=-0,0007521 \\
\mathrm{~b}=0,000000145\end{array}$ & 0,999 & 0,098 & 0,008 \\
\hline & 70 & $\begin{array}{l}a=-0,00105 \\
b=0,000000279\end{array}$ & 0,996 & 0,419 & 0,019 \\
\hline
\end{tabular}




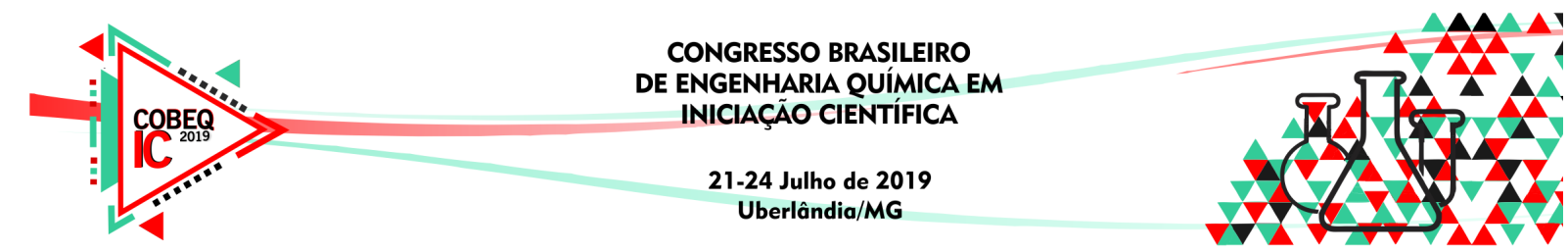

\section{CONCLUSÕES}

Após análise dos dados, pode ser observado que os todos os modelos estudados podem ser empregados no ajuste dos dados experimentais, com significativo grau de credibilidade. Porém, os parâmetros estabelecidos em cada modelo, sugerem que os modelos matemáticos de Page Modificado, Henderson \& Pabis Modificado, Midilli, Verna e Wang \& Singh são os mais adequados por resultarem em valores bem próximos da idealidade, segundo a literatura.

Além disso, observa-se também que quanto maior a temperatura de secagem utilizada, mais rápida a água das folhas evapora, acarretando em porcentagens de umidade mais consistentes. Para as folhas de nim atingirem a umidade de equilíbrio foram necessários 3120 s a $60^{\circ} \mathrm{C}, 2580 \mathrm{~s}$ a $65^{\circ} \mathrm{C}$ e $2280 \mathrm{~s} \mathrm{a} 70^{\circ} \mathrm{C}$.

\section{REFERENCIAS}

BRASIL, R. B. Aspectos botânicos, usos tradicionais e potencialidades de Azadirachta indica (neem). Enciclop. Biosf., Goiânia, v.9, n.17, p.3253, 2013.

EVIN, D. Thin layer drying of Gundelia toumefortii L. FBP, v. 90, p. 323, 2012.

GARCIA, D. C.; SOUZA, A. C. A. B.; PESKE, S. T.; LEMOS, N. M. A secagem de sementes. Cienc. Rural, v.34, p.603, Universidade Federal de Santa Maria. 2004.

GARDUSI, F.; MENDES, L. G.; NOGUEIRA, G. D. R; SILVA, D. I. S.; BARROZO, M. A. S. Secagem da casca da mexerica por irradiação infravermelha e comparação dos compostos bioativos desta in natura e após a secagem. In: X COBEQ IC, 2014.

GONELI, A. L. D.; NASU, A. K.; GANCEDO, R.; ARAÚJO, W. D.; SARATH, K. L. L. Cinética de secagem de folhas de erva baleeira. Rev. Bras. Plantas Med., v. 16, p. 434, 2014.

MARTINEZ, S. S. O nim - Azadirachta indica - Natureza, usos múltiplos, produção. Londrina, IAPAR, 142 p., 2002.

PRATES, M. F. O.; REIS, R. C.; DEVILLA, I. A.; FARIA, R. Q.; LIMA JUNIOR, A. F. Cinética de secagem de folha de Solanum lycocarpum A. St.-Hil (fruta de lobo). Rev. Bras. Plantas Med., v. 14, p. 514, 2012.

SCHMUTTERER H. Insecta, insects. In: The Neem Tree, Schmutterer H (ed). VCH Weinheim; 167-365, 1995.

SIGMA-ALDRICH. Neem (Azadiracta indica). Natural Standard Corporation, 2010.

SINGH, K. K.; TOMAR, A. Neem: An Introduction. In: Neem - A Treatise. Ed. K. K. Singh, Suman Phogat, R. S. Dillon and Alka Tomar. New Delhi: I. K. International Publishing House Pvt. Ltd., 2008. 